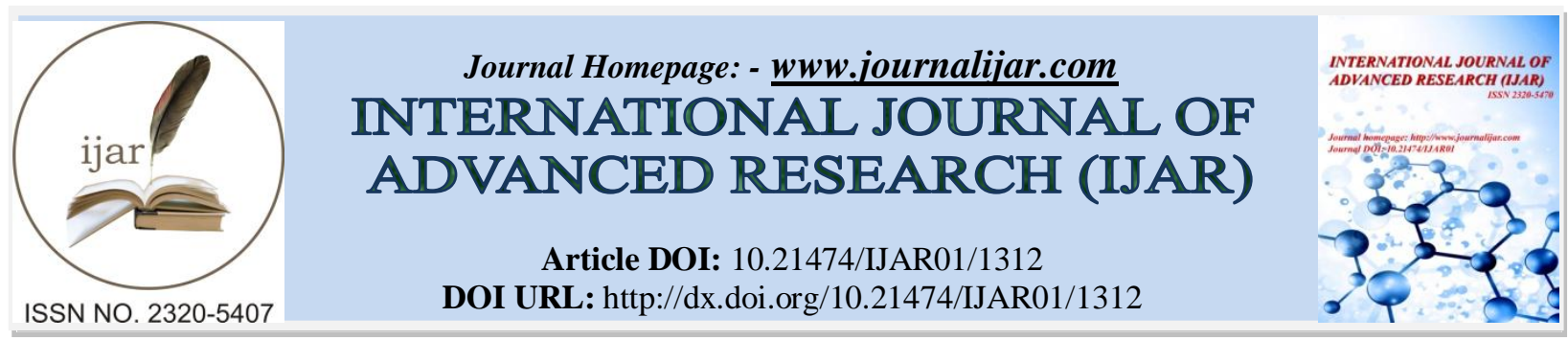

RESEARCH ARTICLE

\title{
DETERMINANTS OF SURGICAL SITE INFECTIONS AFTER BREAST SURGERY; OUR INSTITUTE EXPERIENCE.
}

Rabie Ramadan MD and Ahmed Shaaban MD.

Department of Surgery, Medical Research Institute, Alexandria University.

\section{Manuscript Info}

Manuscript History

Received: 12 June 2016

Final Accepted: 19 July 2016

Published: August 2016

Key words:-

Determinants, Risk Factors, Surgical

Site Infection, Breast Surgery,

Southampton wound scoring system.

\begin{abstract}
Background:- Surgical site infections (SSIs) following breast surgery result in increased length of hospital stay, antibiotic utilization, and morbidity. Understanding SSI risk factors is essential to develop infection prevention strategies and improve surgical outcomes. This study aimed to identify the determinants of SSIs after different breast surgical procedures regarding incidence rate, grades, causative organism(s), risk factors and management at Alexandria Medical Research Institute hospital.

Methods:- The study prospectively included all patients admitted to the department of Surgery, Alexandria Medical Research Institute hospital during the period from May 2014 to April 2015 who were planned for breast surgery. Patients were followed up for 30 days after surgery if no prosthesis was placed during the operation and for one year if prosthesis was placed. The determinants of SSIs regarding incidence rate, grades, causative organism(s), risk factors and management were registered.

Results:- The study included 282 patients of whom 31 (11\%) developed SSIs. All patients with SSI have been detected during the outpatient follow up within the first 3 weeks after surgery except 4 cases; Two cases with implants ( 3 and 6 months after surgery) and 2 cases with expanders ( 7 and 9 months after surgery). Staphylococcus aureus was the most common pathogen $(42 \%)$. Twelve patients $(38.7 \%)$ were readmitted for management of SSI. The incidence of SSI had statistically significant association with age $\geq 60$ years, smoking, diabetes, neoadjuvant chemotherapy, BMI $>35$, type of surgical procedure, prosthesis placement (implant or expander) and seroma formation $(\mathrm{P}=0.003,0.002,0.04,0.03,0.01,0.03,0.02$ and 0.03 respectively).

Conclusion: SSIs after breast surgery are not uncommon complication and can occur after any type of breast surgery. Microbiological diagnosis (culture and sensitivity testing) is recommended for every case with SSI with the use of empirical broad-spectrum antimicrobial coverage until culture results become available. The incidence of SSI had statistically significant association with age $\geq 60$ years, smoking, diabetes, neoadjuvant chemotherapy, BMI >35, type of surgical procedure, prosthesis placement (implant or expander) and seroma formation.
\end{abstract}

Copy Right, IJAR, 2016,. All rights reserved. 


\section{Introduction:-}

Surgical site infection (SSI) is the most common postoperative complication associated with breast surgery (1) and they are major sources of adverse operation-related events in patients undergoing surgery, including increased morbidity, psychological trauma, additional cost and delay of postoperative adjuvant therapies.(2,3) Knowledge of specific risk factors for SSIs is essential in order to create a SSI risk stratification index specific to breast surgery and other types of surgical procedures.(1). The Centers for Disease Control and Prevention (CDC) developed a standard definition of Surgical site infection (SSI) used by most hospital epidemiologists and infection control practitioners worldwide and specifies surveillance for Surgical site infections (SSIs) for 30 days after operation in procedures without prosthesis, and 1 year after operation when a prosthesis is placed.(4) The rate of breast SSIs range from $1 \%$ to $30 \%$, depending on definition of SSIs, type of operation, comorbidities of the patients, time of follow up, Perioperative therapy and reporting institution. It's higher than other clean operations in which the infection rate is less than 5\%. (5-9) Many risk factors for SSI after breast surgery have been reported including older age, obesity, heavy alcohol use, (10-13) smoking, diabetes, malignant tumor, previous open biopsy, $(14,15)$ previous chemotherapy or radiation therapy, (13, 16-20) trainee surgeon responsible for the operation, (21) Seroma development, prolonged duration of drainage after operation, $(13,20)$ immediate reconstruction, and lack of antibiotic prophylaxis at the time of operation. (22-24) This study aimed to identify the determinants of SSIs after different breast surgical procedures regarding incidence rate, grades, causative organism(s), risk factors and management at Alexandria Medical Research Institute hospital.

\section{Patients and methods:-}

The study prospectively included all patients admitted to the department of Surgery, the hospital of Medical Research Institute, University of Alexandria during the period from May 2014 to April 2015 who were planned for different breast surgical procedures. All patients included in this study were subjected to complete history taking stressing on medical history especially diabetes mellitus (DM), Smoking and previous neoadjuvant chemotherapy in cases of breast cancer. Body Mass Index (BMI) for every patient was registered. All patients included in this study recieved $3^{\text {rd }}$ generation cephalosporin antibiotic immediately before the procedure. The types of surgical procedures were registered and patients were followed up for 30 days after surgery if no prosthesis (implant or expander) and up to one year when there was a prosthesis placed during the operation. The incidence of seroma formation after surgery was registered. Prospective detection was used to identify patients who developed SSIs. The grades of SSIs were identified using Southampton wound scoring system. (25) Diagnosis was based on collecting information from clinical outpatient follow up, patients medical records including reviewing of clinical data (symptoms and signs), investigations (laboratory, histopathology, radiological, etc.), microbiological culture and sensitivity results, and medication charts in addition to reviewing the medical records of the infection control team in the hospital. Infections were identified either during the original surgical admission, at readmission to the hospital, or during outpatient follow up of the surgical wound. Causative organisms were recorded from the microbiological reports. Also we registered how these infections were managed. Results were recorded and tabulated using Microsoft Access (Microsoft Corporation, Redmond Washington, USA). Chi-square tests were performed on the data, and p-values were used to evaluate the data for statistical significance.

\section{Results:-}

The study included 282 patients who were admitted to the department of Surgery, the hospital of Medical Research Institute, University of Alexandria during the period from May 2014 to April 2015 who were planned for different breast surgical procedures. Surgical site infections were diagnosed after 31 procedures (11\%). The distribution of the studied patients who had SSIs according to the type of surgical procedure is shown in table I. All patients who had SSI have been detected during the outpatient follow up within the first 3 weeks after surgery except 4 patients; Two patients with implants in whom SSIs had been detected 3 and 6 months after surgery and the other 2 patients with expanders in whom SSIs had been detected 7 and 9 months after surgery. The distribution of patients who had SSIs after breast surgery according to the degree of infection (Southampton wound scoring system) is shown in Table II. Staphylococcus aureus (S.aureus) was the most common pathogen isolated (13 patients (42\%) of all patients), Streptococcus pyogenes was isolated from 5 patients (16.1\%), Escherichia Coli (E. Coli) from 5 patients (16.1\%), Pseudomonas aeruginosa from 4 patients $(12.9 \%)$ and no pathogen was isolated in 4 patients (12.9\%). All patients received antibiotics according to culture and sensitivity results. Twelve patients (38.7\%) out of the 31 who had SSI were readmitted for management of SSI; Seven patients for 2ry suture after debridement, three patients for removal of the infected prosthesis and two patients for debridement. The risk factors studied to evaluate their association with development of SSIs included age, smoking, diabetes, neoadjuvant 
chemotherapy (NAC), BMI, type of surgical procedure, prosthesis placement (implant or expander) and the incidence of Seroma formation. The incidence of SSI had statistically significant association with age $\geq 60$ years, smoking, diabetes, NAC, BMI $>35$, type of surgical procedure, prosthesis placement (implant or expander) and seroma formation $(\mathrm{P}=0.003,0.002,0.04,0.03,0.01,0.03,0.02$ and 0.03 respectively). The risk factors studied to evaluate their association with development of SSIs were summarized in table (III). Figures 1 and 2 showed examples of patients that had SSI and included in the studied patients.

Table I:- Distribution of patients who had SSIs according to the type of the surgical procedure submitted.

\begin{tabular}{|c|c|c|}
\hline Surgical Procedure & $\begin{array}{l}\text { Number of patients submitted to this } \\
\text { procedure }(\%)\end{array}$ & $\begin{array}{l}\text { Number of patients submitted to } \\
\text { this procedure and developed SSIs } \\
(\%)\end{array}$ \\
\hline Lumpectomy & $82(29.1 \%)$ & $3(9.7 \%)$ \\
\hline MDE & $22(7.8 \%)$ & $2(6.4 \%)$ \\
\hline Nipple reconstruction & $8(2.8 \%)$ & - \\
\hline Lipofilling & $19(6.7 \%)$ & - \\
\hline C B S & $26(9.2 \%)$ & $3(9.7 \%)$ \\
\hline Mastectomy & $80(28.3 \%)$ & $13(42 \%)$ \\
\hline Reduction mammaplasty & $14(5.1 \%)$ & $3(9.7 \%)$ \\
\hline TRAM & $4(1.4 \%)$ & $2(6.4 \%)$ \\
\hline LD & $10(3.6 \%)$ & - \\
\hline Implant & $9(3.2 \%)$ & $2(6.4 \%)$ \\
\hline Expander & $8(2.8 \%)$ & $3(9.7 \%)$ \\
\hline TOTAL & $282(100 \%)$ & $31(100 \%)$ \\
\hline
\end{tabular}

MDE: Major Duct Excision CBS: Conservative Breast Surgery

TRAM: Transverse Rectus Abdominis Myocutaneous Flap

LD: Latissmus Dorsi Myocutaneous Flap

Table II:- Distribution of patients who had SSI after breast surgery according to the degree of infection according to Southampton wound scoring system. ${ }^{(25)}$

\begin{tabular}{|c|c|}
\hline \multicolumn{2}{|c|}{ Number of patients who developed this degree of SSIs (\%) } \\
\hline SOUTHAMPTON SCORE \\
\hline IIB & $\mathbf{4 ( 1 2 . 9 \% )}$ \\
\hline IIC & $\mathbf{5 ( 1 6 . 1 \% )}$ \\
\hline IIIB & $\mathbf{3 ( 9 . 7 \% )}$ \\
\hline IIIC & $\mathbf{4 ( 1 2 . 9 \% )}$ \\
\hline IIID & $\mathbf{3 ( 9 . 7 \% )}$ \\
\hline IVA & $\mathbf{5 ( 1 6 . 1 \% )}$ \\
\hline IVB & $\mathbf{3 ( 9 . 7 \% )}$ \\
\hline V & $\mathbf{4 ( 1 2 . 9 \% )}$ \\
\hline TOTAL & $\mathbf{3 1}(\mathbf{1 0 0 \%})$ \\
\hline
\end{tabular}


Table III:- Risk Factors associated with the development of SSIs after Breast Surgery.

\begin{tabular}{|l|l|l|l|}
\hline Risk factor & $\begin{array}{l}\text { No. of of } \\
\text { patients } \\
\text { having this } \\
\text { risk factor }\end{array}$ & $\begin{array}{l}\text { No. of patients having } \\
\text { this risk factor and } \\
\text { developing SSIs }\end{array}$ & P-value \\
\hline Age(y) & & & \\
\hline$<60$ & $188 / 282$ & $9 / 31$ & $\mathbf{0 . 0 0 3}$ \\
\hline$\geq 60$ & $94 / 282$ & $22 / 31$ & $\mathbf{0 . 0 0 2}$ \\
\hline Smoking & $18 / 282$ & $13 / 31$ & $\mathbf{0 . 0 4}$ \\
\hline Diabetes & $54 / 282$ & $11 / 31$ & $\mathbf{0 . 0 3}$ \\
\hline Neoadjuvant Chemotherapy & $32 / 282$ & $14 / 31$ & $\mathbf{0 . 0 1}$ \\
\hline BMI & & & \\
\hline$\leq 35$ & $102 / 282$ & $7 / 31$ & $\mathbf{0 . 0 3}$ \\
\hline$>35$ & $180 / 282$ & $24 / 31$ & \\
\hline Type of Surgery & & & \\
\hline Lumpectomy & $82 / 282$ & $3 / 31$ & \\
\hline MDE & $22 / 282$ & $2 / 31$ & \\
\hline CBS & $26 / 282$ & $3 / 31$ & \\
\hline Mastectomy & $80 / 282$ & $13 / 31$ & \\
\hline Reduction mammaplasty & $14 / 282$ & $3 / 31$ & $\mathbf{0 . 0 2}$ \\
\hline TRAM & $4 / 282$ & $2 / 31$ & $\mathbf{0 . 0 3}$ \\
\hline Implant & $9 / 282$ & $2 / 31$ & \\
\hline Expander & $8 / 282$ & $3 / 31$ & $5 / 31$ \\
\hline Prosthesis insertion & $17 / 282$ & $16 / 31$ & \\
\hline Seroma formation & $38 / 282$ & & \\
\hline
\end{tabular}




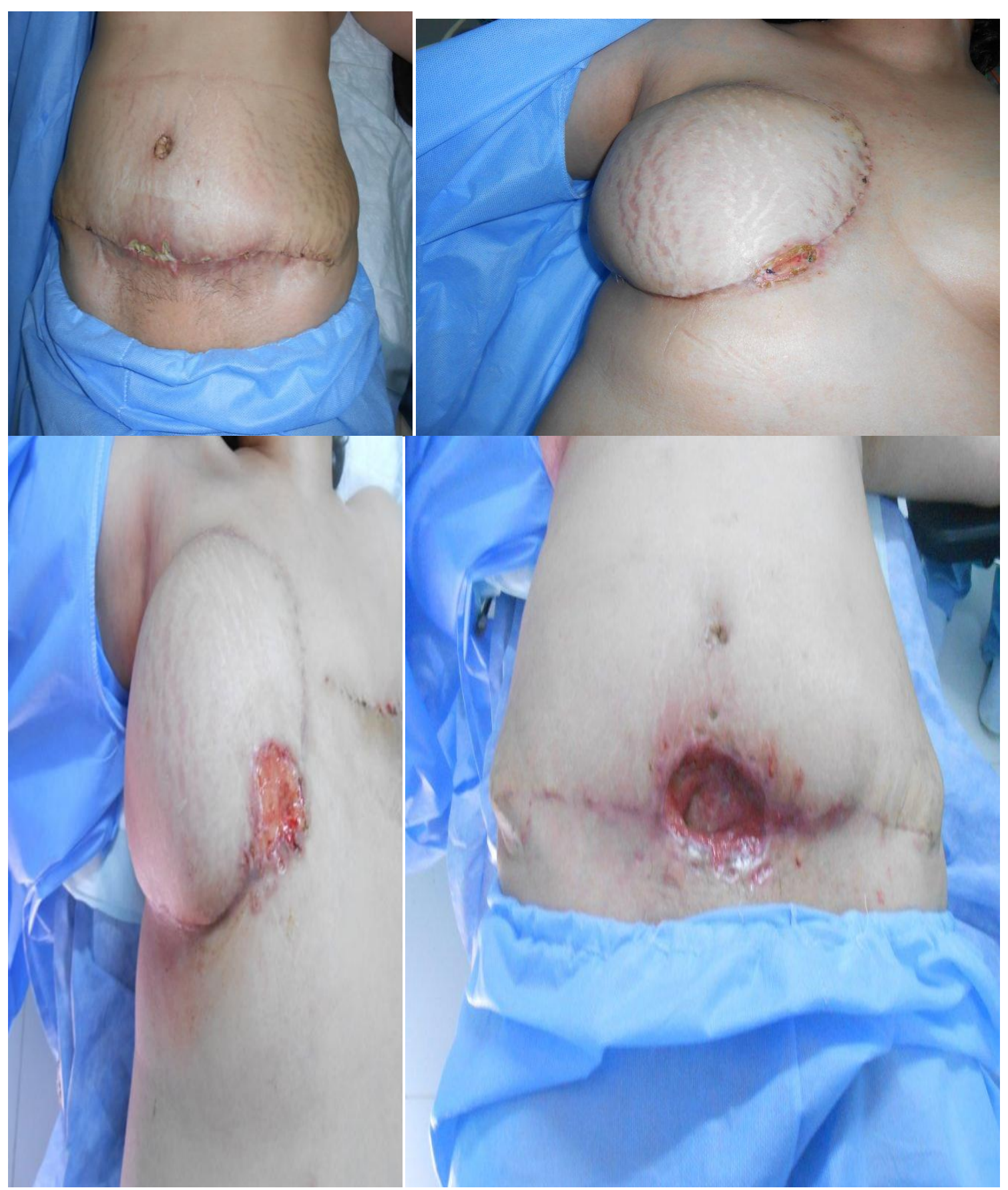

Figure 1:- 47 years old female with right breast cancer submitted for mastectomy with immediate reconstruction with TRAM; She developed SSI at both the breast and abdominal wounds; both wounds were debrided and left to heal by $2^{\text {nd }}$ intention healing. 


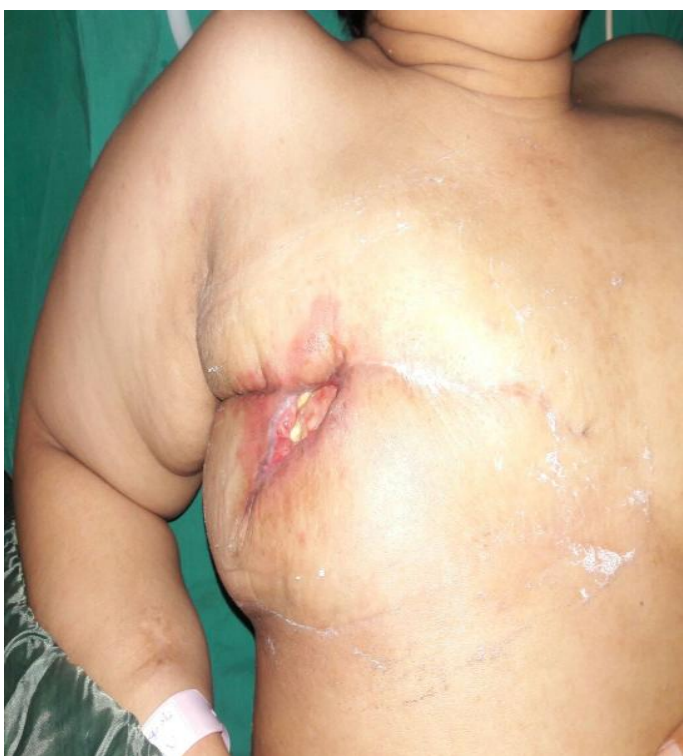

A

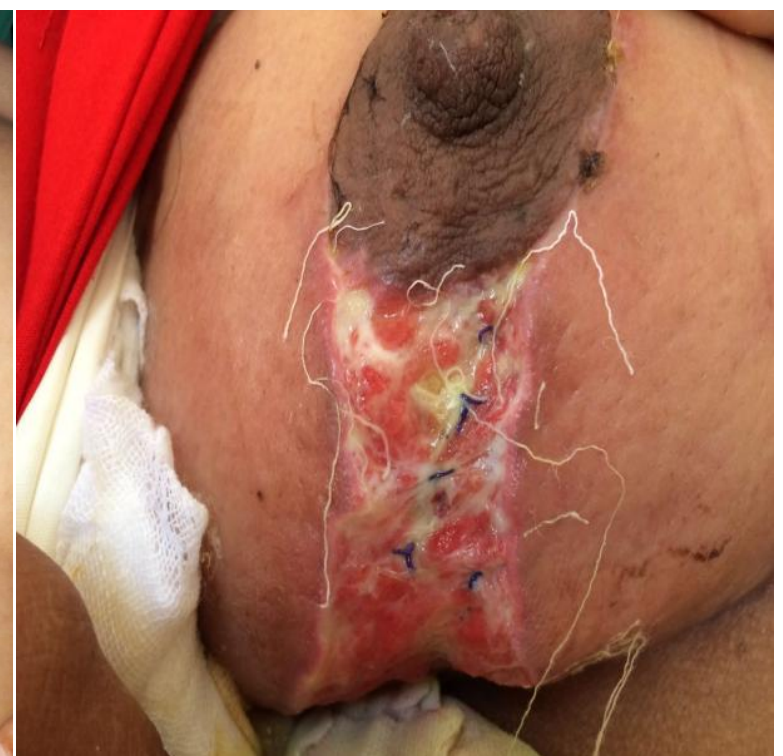

B

Figure 2:- Two patients with SSIs; A: MRM for right breast cancer with fish-tail plasty; B: Therapeutic mammaplasty for right breast cancer

\section{Discussion:-}

Understanding surgical site infection (SSI) risk factors after breast operation is essential to develop infectionprevention strategies and improve surgical outcomes. (26) We reported higher rate of SSIs (11\%) than reported by Degnim et al. (2.7\%) (27), Leinung et al. (4.5\%) (28), Olsen et al. (4.7\%) (29) and Abeer Omar et al. (2.3\%)(30); but lower than that reported by Vilar-Compte et al. (18.9\% (30) Abeer Omar et al (30) attributed their lower rate of SSIs in their study to the concept of decreasing postoperative length of hospital stay so the follow up of the patient is mainly carried out on an outpatient basis. During outpatient visits, when the SSI develops and requires no readmission, surgeons may not document the infection in the patient's records and may not request microbiological sampling of the wound. This is primarily due to fear of medical malpractice claims or negligence especially in a surgery classified as a clean one like breast surgery.(30) We considered this bias is not present in our study for two reasons: the first: we have taken our data from two sources: files of Surgery department and files of infection control unit, the second; Our Institute specialized breast clinic has its own files in which all data are registered by residents who have fixed modules to be filled with follow up data and photos. In our study; Twelve patients (38.7\%) out of the 31 who had SSI were readmitted for management of SSI; Seven patients for 2ry suture after debridement, three patients for removal of the infected prosthesis and two patients for debridement while In another study;(12) Sixty two percent were readmitted for management of SSI. We attributed the lower rate of readmission in our study to that only 12 patients $(38.7 \%$ ) out of the 31 who had SSIs had severe grades of SSIs (Grade IV and V). In the present study; despite the fact that S. aureus was the primary pathogen isolated from SSIs (42\%); Gram negative bacteria (E. Coli and Pseudomonas aeruginosa) were isolated in $29 \%$ of cases representing a significant finding. Other studies reported the same results (32). However, Mukhtar et al. (33) reported Gram negative bacteria as the most common isolated pathogens. These findings support the importance of the use of empirical broadspectrum antimicrobial (not only targeting S.aureus) coverage until culture results become available. In some articles, it was proposed that administrating the antibiotics would significantly decrease the risk of SSI only in high risk patients and its application is not essential for all cases (34). To omit the effect of this factor; All patients who were submitted for any procedure recieved $3^{\text {rd }}$ generation Cephalosporin antibiotic immediately before the procedure as the cost of treating the SSI in patients without prophylaxis has been higher than the cost of the antibiotic prophylaxis for breast cases, which showed that this was a cost effective strategy for reducing the SSI. in our study; thirteen patients (42\%) out of the 31 who had SSIs were submitted to mastectomy which is similar to the results of another study (35) which concluded that mastectomy was associated with increased rate of SSI incidence when compared to other types of breast surgeries which may be attributed to the interruption of lymphatic drainage and duration of drainage which may participate in higher possibility of SSI after the mastectomy compared to other similar breast operations. We agreed with these explanations but we can add that mastectomy is still the commonest major submitted procedure (28.3\%). In the present study; We concluded smoking as a significant risk 
factor for developing SSIs after breast surgery which matched with several articles which had mentioned smoking as a considerable independent risk factor for postoperative SSI due to its effects on preventing the adequate blood supply and the negative effect of nicotine on wound healing which increases the SSI rate. $(36,37)$. Based on the study of Sorensen et al., smoking could be considered as a prognostic parameter of SSI after breast surgeries, which might result in skin flap necrosis, epidermolysis and delayed wound healing (37). In one meta-analysis performed in 2012, different variables were studied to assess their relation with increased SSI after breast surgeries (39). According to eight articles studied in the mentioned meta-analysis; increased age, obesity, DM, presence of postoperative seroma and selected operative procedure were the major predictive parameters of SSI which are similar to our results which concluded age $\geq 60$ years, diabetes, BMI $>35$, type of surgical procedure and seroma formation as statistically significant risk factors for SSI after breast surgery. The presence of these factors in patients should be evaluated before the surgery to be managed and controlled to decrease the possibility of the SSI occurrence. We concluded increased risk of SSIs with immediate placement of prosthesis (implant or expander) after mastectomy $(\mathrm{P}=0.02)$ which is similar to many studies $(40-42)$; but really we cannot conclude whether the implant alone is responsible for the increased risk of SSI, or if it is the combination of mastectomy followed by immediate placement of prosthesis that confers increased risk of SSI as we had no control group with mastectomy with delayed prosthesis insertion. Finally; we concluded that receiving NAC had statistically significant association with the incidence of SSI which is supported by the results of many recent studies. (13, 16-20) but differs from the results of Olsen et al (35) who were unable to detect any association between SSI and NAC.

\section{Conclusion and recommendations:-}

SSIs after breast surgery are not uncommon complication and can occur after any type of breast surgery. Microbiological diagnosis (culture and sensitivity testing) is recommended for every case with SSI with the use of empirical broad-spectrum antimicrobial (not only targeting S. aureus) coverage until culture results become available. The incidence of SSI had statistically significant association with Age $\geq 60$ years, Smoking, Diabetes, Neoadjuvant Chemotherapy, BMI >35, Type of surgical procedure, Prosthesis placement (Implant or expander) and Seroma formation. The knowledge about these potential risk factors associated with breast SSI can be beneficial in better managing, controlling and reducing the occurrence of the SSI and developing the quality of patients' treatments. We recommend further studies with larger volume of cases to confirm the results and to study more risk factors.

\section{Acknowledgement:-}

We would like to thank the Infection Control Team in our institute who supplied us with data which helped us to conduct this work.

\section{Conflict of Interest:-}

The authors declare no conflict of interest.

\section{References:}

1. W. A. B. Felippe, G. L. Werneck, and G. Santoro-Lopes, "Surgical site infection among women discharged with a drain in situ after breast cancer surgery," World Journal of Surgery 2007; 31(12): 2293-9.

2. Hughes WT, Flynn PM, Williams BG. Nosocomial infections in patients with neoplastic diseases. In: Mayhall CG, editor. Hospital Epidemiology and infections control. 2nd ed. Philadelphia: Lippincott Williams \& Wilkins; 1999: 767-71.

3. Robstein C, Ferguson R, Cummings KM, et al. Determinants of clean surgical wound infections for breast procedures at an oncologic centre. Infect Control Hosp Epidemiol 1992; $79: 314-7$.

4. Mangram AJ, Horan TC, Pearson ML, et al. Guideline for prevention of surgical site infection, 1999. Infect Control Hosp Epidemiol 1999; 20: 247-78.

5. Gaynes RP, Culver DH, Horan TC, et al. Surgical site infections (SSI) rates in the United States, 1992e1998: the national nosocomial infections surveillance system basic SSI risk index. Clin Infect Dis 2001 Sep 1; (Suppl. 2):S69-77. 33

6. Hall JC, Hall JL. Antibiotic prophylaxis for patients undergoing breast surgery. J Hosp Infect 2000; 46:165-70.

7. Canavese G, Catturich A, Vecchio $C$, et al. Surgical complications related to peri-operative adjuvant chemotherapy in breast cancer: results of a prospective, controlled, randomized clinical trial. Eur J Surg Oncol 1997; 23: 10-2. 
8. National Nosocomial Infections Surveillance System. National nosocomial infections surveillance (NNIS) system report, data summary from January 1992 through June 2004, issued October 2004. Am J Infect Control 2004; 32:470-85.

9. K. R. Wedgwood, E. A. Benson, and B. Hogbin, "Non-tumour morbidity and mortality after modified radical mastectomy, " Annals of the Royal College of Surgeons of England1992; 74(5): 314-7.

10. Witt A, YavuzD, Walchetseder C, et al. Preoperative core needle biopsy as an independent risk factor for wound infection after breast surgery. Obstet Gynecol 2003; 101: 745-50.

11. Rotstein C, Ferguson R, Cummings KM, et al. Determinants of clean surgical wound infections for breast procedures at an oncology center. Infect Control Hosp Epidemiol 1992; 13: 207-14.

12. Vinton AL, Traverso LW, Zehring RD. Immediate breast recon- struction following mastectomy is as safe as mastectomy alone. Arch Surg 1990; 125: 1303-8.

13. Sorensen LT, Horby J, Friis E, et al. Smoking as a risk factor for wound healing and infection in breast cancer surgery. Eur J Surg Oncol 2002; 28: 815-820.

14. Vilar-Compte D, Jacquemin B, Robles-Vidal C, Volkow P . Surgical site infections in breast surgery: Casecontrol study. World J Surg 2004; 28: 242-6.

15. Beatty JD, RobinsonGV, Zaia JA, et al. A prospective analysis of nosocomial wound infection aftermastectomy. Arch Surg 1983; 118: 1421-4.

16. Nieto A, Lozano M, Moro MT, et al. Determinants of wound infections after surgery for breast cancer. Zentralbl Gynakol 2002; 124: 429-33.

17. Say CC, Donegan W. A biostatistical evaluation of complications from mastectomy. Surg Gynecol Obstet 1974; 138: 370-6.

18. Krueger EA,Wilkins EG, StrawdermanM, et al. Complications and patient satisfaction following expander/implant breast reconstruction with and without radiotherapy. Int J Radiat Oncol Biol Phys 2001; 49: 713-21.

19. Nahabedian MY, Tsangaris T, Momen B, Manson PN. Infectious complications following breast reconstruction with expanders and implants. Plast Reconstr Surg 2003;112: 467-76.

20. Vandeweyer E, Deraemaecker R, Nogaret JM, Hertens D. Immediate breast reconstruction with implants and adjuvant chemotherapy: a good option? Acta Chir Belg 2003; 103: 98-101.

21. Lefebvre D, Penel N, Deberles MF, Fournier C. Incidence and surgical wound infection risk factors in breast cancer surgery. Presse Med 2000; 29: 1927-1932.

22. Hayes JA, Bryan RM. Wound healing following mastectomy. Aust NZ J Surg 1984; 54: $25-7$.

23. Platt R, Zucker JR, Zaleznik DF, et al. Prophylaxis against wound infection following herniorrhaphy or breast surgery. J In- fect Dis 1992; 166: 556-60.

24. Cunningham M, Bunn F, Handscomb K. Prophylactic antibiotics to prevent surgical site infection after breast cancer surgery. Cochrane Database Syst Rev 2006; Apr 19; (2): CD005360.

25. Bailey IS, Karran SE, Toyn K. Community surveillance of complications after hernia surgery. BMJ 1992; 304(6825): 469-71.

26. Margaret A. Olsen, PhD, MPH , Mellani Lefta, et al. Risk Factors for Surgical Site Infection Following Major Breast Surgery. J Am Coll Surg. 2008 September; 207(3): 326-35.

27. C. Degnim, A. D. Throckmorton, S. Y. Boostrom et al., "Surgical site infection after breast surgery: impact of 2010CDC reporting guidelines," Annals of Surgical Oncology 2012; 19: 4099-103.

28. S. Leinung, M. Sch?nfelder, K. J. Winzer et al., "Wound infection and infection-promoting factors in breast cancer surgery - a prospective multicenter study on quality control, "Zentralblatt für Chirurgie 2005; 130(1):16-20.

29. M. A. Olsen, S. Chu-Ongsakul, K. E. Brandt, J. R. Dietz, J. Mayfield, and V. J. Fraser, "Hospital-associated costs due to surgical site infection after breast surgery, " Archives of Surgery2008; 143(1):53-60.

30. Abeer A. Omar and Haifaa H. Al-Mousa. Surgical Site Infection Complicating Breast Cancer Surgery in Kuwait. ISRN Preventive Medicine,Volume 2013, Article ID 295783, Page 2.

31. D.Vilar-Compte, S. Rosales,N.Hernandez-Mello, E.Maafs, and P. Volkow, "Surveillance, control, and prevention of surgical site infections in breast cancer surgery: a 5-year experience, " American Journal of Infection Control 2009; 37(8): 674-9.

32. D. Throckmorton, L. M. Baddour, T. L. Hoskin, J. C. Boughey, and A. C. Degnim, "Microbiology of surgical site infections complicating breast surgery," Surgical Infections 2010; 11(4): 355-9.

33. R. A. Mukhtar, A. D. frockmorton, M. D. Alvarado et al., "Bacteriologic features of surgical site infections following breast surgery,” American Journal of Surgery 2009; 198(4): 529-31. 
34. Nicolas P, Yazdan Y, Marie-Pierre C, et al. Prevention of surgical site infection after breast cancer surgery by targeted prophylaxis antibiotic in patients at high risk of surgical site infection. J Surg Oncol. 2007; 96: 124-9.

35. Olsen MA, Chu-Ongsakul S, Brandt KE, et al. Hospital-associated costs due to surgical site infection after breast surgery. Arch Surg. 2008;143: 53-60.

36. Bertin ML, Crowe J, Gordon SM. Determinants of surgical site infection after breast surgery. Am J Infect Control. 1998; 26: 61-5.

37. Zimmermann-Nielsen E, Dahl M, Graversen H. Effects of tobacco smoking on the incidence of flap necrosis after mastectomy. Ugeskr Laeger. 1997;159: 4974-6.

38. Sørensen L, Hørby J, Friis E, et al. Smoking as a risk factor for wound healing and infection in breast cancer surgery. Eur J Surg Oncol. 2002; 28: 815-20.

39. Xue D, Qian C, Yang L, et al. Risk factors for surgical site infections after breast surgery: a systematic review and meta-analysis. Eur J Surg Oncol. 2012; 38: 375-81.

40. Nahabedian MY, Tsangaris T, Momen B, Manson PN. Infectious complications following breast reconstruction with ex-panders and implants. Plast Reconstr Surg 2003;112: 467-76.

41. Vandeweyer E, Deraemaecker R, Nogaret JM, Hertens D. Immediate breast reconstruction with implants and adjuvant chemotherapy: a good option? Acta Chir Belg 2003; 103: 98-101.

42. Van Geel AN, Contant CM, Wai RT, et al. Mastectomy by inverted drip incision and immediate reconstruction: data from510 cases. Ann Surg Oncol 2003; 10: 389-95. 